\title{
Consensus of High-Order Linear Multiagent Systems with Multitype Switching Topologies Based on the Dynamic Dwell Time Approach
}

\author{
Yaxiao Zhang, Yangzhou Chen, and Xiaojun Qu \\ Beijing Key Laboratory of Transportation Engineering, College of Metropolitan Transportation, Beijing University of Technology, \\ Beijing 100124, China
}

Correspondence should be addressed to Yaxiao Zhang; zhangyaxiao@emails.bjut.edu.cn

Received 21 October 2015; Accepted 8 December 2015

Academic Editor: Weiguo Xia

Copyright (c) 2016 Yaxiao Zhang et al. This is an open access article distributed under the Creative Commons Attribution License, which permits unrestricted use, distribution, and reproduction in any medium, provided the original work is properly cited.

\begin{abstract}
This paper investigates the consensus problem of high-order continuous-time linear multiagent systems (LMASs) with multitype switching topologies which include both consensusable and unconsensusable communication topologies. A linear transformation is introduced, which equivalently transforms the consensus problem into the stability problem of a corresponding switched system, along with a necessary and sufficient condition to analyze the consensus problem. This paper is aimed at studying the impact of a switching rule on communication topologies for consensus of LMASs. Based on the dynamic dwell time method, a sufficient condition is derived for consensus of LMASs. It is shown that, with switching signals that satisfy this switching rule, LMASs can achieve consensus under directed switching communication topologies. A numerical example is provided to illustrate the effectiveness of the theoretical results.
\end{abstract}

\section{Introduction}

In the past few decades, the study of distributed coordination control of multiagent systems has been a hot topic due to the extensive applications in various specialized areas, including flocking [1], formation control [2], the cooperative control of unmanned air vehicles [3], and communication control of large scale networks [4]. Consensus, as one of the most important subjects in distributed coordination control, means that, by appropriate control protocols, multiple agents can reach agreement on certain quantities of interest, such as velocity, position, or heading direction.

Numerous significant theoretical results in the literature have dealt with the consensus problem of multiagent systems in the framework of first-order/second-order state space [5-7]. However, due to the complexity and diversity of agents in the real world, high-order dynamics should be considered. Reference [8] investigated a general high-order model for linear multiagent systems (LMASs) under a fixed communication topology and presented some consensus criteria. The impact of the agent dynamic structure and the communication topology on consensusability was pointed out in [9]. Reference [10] derived some necessary and sufficient conditions for ensuring consensus of high-order system and introduced the concept of consensus regions. In [11], consensus criteria and consensus functions were obtained, and the design of feedback gain matrix in protocol was provided.

Moreover, link failure and creation occur frequently due to, for instance, the sensors limited communication range, transmission failures, and certain obstacles. Therefore, the study of the consensus of high-order LMASs under switching topologies is more practical. Under the assumption that the communication topologies are undirected, a sufficient condition for consensus was derived in [12] with a constraint that the communication topologies are required to be "frequently connected." In [13], the communication topology condition was extended to "jointly connected." Based on digraphs, [14] relaxed the communication topology conditions in $[12,13]$ and pointed out the fact that the "uniformly jointly quasistrongly connected" topology is critical for LMASs to achieve consensus. 
It is well known that there are three key factors for consensus of LMAS under fixed topology: dynamic structure of agents, communication topology of LMASs, and control protocol. Further, switching signals on communication topologies play a key role in achieving consensus for LMASs under switching topologies. When dynamic structures of agents and possible communication topologies are given, one can study the relationship between the consensus and the switching rule of communication topologies. A natural question which arises is with what switching signal can the LMASs achieve consensus? Literature addressing this concern has already been published. For instance, by using the average dwell time method, [15] chose a class of signals to deal with a synchronization problem of a nonlinear multiagent system under undirected switching topologies. The topology condition was extended to the digraph in $[16,17]$. However, switching signals based on the average dwell time method are "slow switching" and are too limited to precisely depict the sufficient condition for consensus. This limitation brings difficulties to the analysis of consensus conditions. To overcome this drawback, a switching rule based on the dynamic dwell time method [18] was introduced in [19] with an assumption that all possible communication topologies are consensusable.

However, in practice, the possible communication topologies of LMASs are not always consensusable. Thus, this paper analyzes the consensus problem for high-order LMASs under multitype switching topologies. It is aimed at exploring a switching rule, such that, with a class of switching signals satisfying this rule, consensus can be achieved by LMASs. Compared with the existing works, the contributions of the paper are summarized as follows. (1) Communication topology conditions in [19] are extended to include both consensusable and unconsensusable communication topologies. (2) Compared with the switching rule based on the average dwell time method in $[16,17]$, a switching rule based on the dynamic dwell time method is less conservative and more flexible; in addition, it allows more numbers of switching signals.

An outline of this paper is organized as follows. In Section 2, some basic concepts are described and the problem to be studied is formulated. A linear transformation is presented along with a lemma to describe the equivalent relation between the consensus problem and the stability problem of a corresponding switched system in Section 3. A sufficient condition based on the dynamic dwell time method for the consensus of LMASs is established in Section 4. A numerical example is provided in Section 5. Conclusions are drawn in Section 6.

\section{Problem Description}

Consider a system consisting of $N$ agents, labelled 1 through

$N$, and the dynamic of the $i$ th agent is

$$
\begin{aligned}
& \dot{x}_{i}(t)=A x_{i}(t)+B u_{i}(t), \\
& x_{i}(0)=x_{i 0},
\end{aligned}
$$

where $x_{i} \in \mathbb{R}^{n}$ is the state variable of agent $i, u_{i} \in \mathbb{R}^{m}$ is its control input, $\mathscr{I}=\{1, \ldots, N\}$ is the index set of agents, and $A \in \mathbb{R}^{n \times n}$ and $B \in \mathbb{R}^{n \times m}$ are constant matrices.

The communication topology (hereafter referred to as topology) of system (1) is expressed by a weighted digraph $\mathcal{N}(t)=\{V, E(t), W(t)\}$, where the $i$ th element of the vertex set $V=\left\{v_{1}, \ldots, v_{N}\right\}$ represents agent $i$, and the element $\left(v_{i}, v_{j}\right)$ of the time-varying edge set $E(t) \subseteq V \times V$ denotes the communication link from agents $i$ to $j$. Let $N_{i}(t)=\left\{v_{j} \in\right.$ $\left.V,\left(v_{j}, v_{i}\right) \in E(t)\right\}$ stand for the neighbor set of agent $i$, and weight matrix $W(t)=\left[w_{i j}\right]_{N \times N}$, where $w_{i j}$ is a scalar which represents the coupling strength of information transmitting from agents $j$ to $i, i, j \in \mathscr{I}$.

Using a protocol [5],

$$
u_{i}(t)=K \sum_{j \in N_{i}(t)} w_{i j}(t)\left(x_{j}(t)-x_{i}(t)\right), \quad i \in \mathscr{I},
$$

where $K \in \mathbb{R}^{m \times n}$ is a feedback gain matrix.

Definition 1. System (1) achieves consensus via protocol (2) if, for any initial state, there is $\lim _{t \rightarrow \infty}\left\|x_{i}(t)-x_{j}(t)\right\|=0, i, j \in$ $\mathscr{I}$

Herein $\|\cdot\|$ denotes the Euclidean norm of a vector.

Definition 2 (see [19]). A time-invariant topology $\mathcal{N}$ is called consensusable topology of system (1), if there exists a protocol in form (2) such that system (1) achieves consensus. Otherwise, topology $\mathcal{N}$ is unconsensusable.

For instance, a topology in which there exists a spanning tree is consensusable.

Due to various reasons, such as the sensors limited communication range, transmission failures, and obstacles, the communication links between agents may disconnect or reconnect and result in time-varying topology $\mathcal{N}(t)$. Without loss of generality, suppose there are finite topologies of system (1); that is, $\mathcal{N}(t) \in \mathscr{N} \mathscr{A}=\left\{\mathcal{N}^{l}, l \in \mathfrak{M}\right\}, \mathfrak{M}=\{1, \ldots, M\}$. Within $\mathscr{N} \mathscr{A}$, there are $M_{1}$ consensusable topologies, that is, set $\mathscr{N} \mathscr{C}=\left\{\mathscr{N}^{l}, l \in \mathfrak{M}^{-}\right\}$, and $M_{2}$ unconsensusable topologies, that is, set $\mathcal{N} \mathscr{U}=\left\{\mathscr{N}^{l}, l \in \mathfrak{M}^{+}\right\}$, where $\mathfrak{M}=$ $\mathfrak{M}^{-} \cup \mathfrak{M}^{+}$and $\mathscr{N} \mathscr{A}=\mathscr{N} \mathscr{C} \cup \mathcal{N} \mathscr{U}$. Next, we consider a case which satisfies the following assumption.

Assumption 3. Topology $\mathcal{N}(t)$ switches within set $\mathcal{N} \mathscr{A}=$ $\left\{\mathscr{N}^{l}, l \in \mathfrak{M}\right\}$, where $\mathscr{N}^{l}, l \in \mathfrak{M}^{-}$is consensusable, while $\mathscr{N}^{l}, l \in \mathfrak{M}^{+}$is unconsensusable.

Via protocol (2), system (1) can be expressed by the switched system as follows:

$$
\dot{x}(t)=\left(I_{N} \otimes A-L_{\sigma(t)} \otimes\left(B K_{\sigma(t)}\right)\right) x(t), \quad t \in[0, \infty),
$$

where $x=\left[\begin{array}{lll}x_{1}^{T} & \cdots & x_{N}^{T}\end{array}\right]^{T} \in \mathbb{R}^{N n}, x(0)=x_{0}$ is initial state, $I_{N}$ is the identity matrix of order $N, \otimes$ is the Kronecker product of matrices, $\sigma(t):[0,+\infty) \rightarrow \mathfrak{M}$ is a piecewise constant function which describes the switching law of topology $\mathcal{N}(t)$; namely, $\mathcal{N}(t)=\mathcal{N}^{l} \Leftrightarrow \sigma(t)=l, l \in \mathfrak{M}$. Suppose 
that there is finite switching for $\mathcal{N}(t)$ within any finite time interval. For any given $\sigma(t)$, define the switching sequence $\left\{\left(t_{0}, l_{0}\right),\left(t_{1}, l_{1}\right), \ldots,\left(t_{r}, l_{r}\right), \ldots, \mid l_{r} \in \mathfrak{M}, r=0,1, \ldots\right\}$, where $\sigma\left(t_{r}\right)=l_{r}$; that is, the topology of LMAS (1) is $\mathcal{N}^{l_{r}}$ when $t \in\left[t_{r}, t_{r+1}\right) . L_{\sigma(t)} \in \mathbb{R}^{N \times N}$ is the weighted Laplacian matrix induced by the topology $\mathcal{N}(t)=\left\{N_{i}^{\sigma(t)}, i \in \mathscr{I}\right\}$, and its entries are defined by

$$
l_{i j}^{\sigma(t)}=\left\{\begin{array}{ll}
\sum_{k=1, k \neq i}^{N} w_{i k}^{\sigma(t)}, & j=i \\
-w_{i j}^{\sigma(t)}, & j \neq i,
\end{array} \quad i, j \in \mathscr{I}, j \in N_{i} .\right.
$$

It is generally known that the switching signals of topologies have great impact on the consensus of LMASs. If LMASs follow an incorrect switching signal, consensus cannot be achieved even though $\mathcal{N}(t)$ switches within consensusable topologies. Conversely, if LMASs follow a correct switching signal, consensus can be achieved even if $\mathcal{N}(t)$ switches within unconsensusable topologies. We aim to explore under what condition for the switching signal $\sigma(t)$ can system (1) achieve consensus via protocol (2) with topology $\mathcal{N}(t)$ satisfying Assumption 3. In order to depict the switching signal $\sigma(t)$, we introduce the definition of dwell time.

Definition 4 (see [20]). Let $\left\{t_{k}, k=0,1,2, \ldots\right\}$ be the set of switching instants of a given switching signal $\sigma(t)$, where $t_{0}=$ 0. $\tau_{l}\left(t_{k}\right)=t_{k+1}-t_{k}$ is called the dwell time of system (1) under topology $\mathcal{N}^{l}, l=\sigma\left(t_{k}\right)$.

Next, by a linear transformation, we equivalently transform the consensus problem into an asymptotic stability problem of a corresponding switched system.

\section{Linear Transformation and Consensus Analysis}

Consider the following linear transformation [11]:

$$
\bar{x}=H x, \quad H=:\left[\begin{array}{c}
\widetilde{H}_{0} \\
\mathbf{1}_{N}^{T}
\end{array}\right] \otimes I_{n},
$$

where $\mathbf{1}_{N}^{T}=\left[\begin{array}{llll}1 & 1 & \cdots & 1\end{array}\right]$ is $N$ dimensional vector, $\bar{x}=$ $\left[\begin{array}{llll}\bar{x}_{1}^{T} & \ldots & \bar{x}_{N-1}^{T} & \bar{x}_{N}^{T}\end{array}\right]^{T}$, and $\widetilde{H}_{0}$ is $(N-1) \times N$ matrix defined by

$$
\widetilde{H}_{0}=\left[\begin{array}{ccccc}
1 & -1 & 0 & \cdots & 0 \\
0 & 1 & -1 & \cdots & 0 \\
\vdots & \vdots & \vdots & \ddots & \vdots \\
0 & \cdots & 0 & 1 & -1
\end{array}\right]
$$

The inverse matrix of $H$ can be worked out as follows:

$$
H^{-1}=:\left[\begin{array}{ll}
\widehat{H}_{0} & N^{-1} \mathbf{1}_{N}
\end{array}\right] \otimes I_{n}
$$

where $\widehat{H}_{0}$ is $N \times(N-1)$ matrix given by

$$
\widehat{H}_{0}=\frac{1}{N}\left[\begin{array}{cccc}
N-1 & N-2 & \cdots & 1 \\
-1 & N-2 & \cdots & 1 \\
\vdots & \vdots & \ddots & \vdots \\
-1 & -2 & \cdots & 1 \\
-1 & -2 & \cdots & -(N-1)
\end{array}\right] .
$$

After the linear transformation (5), system (3) is transformed into the following system:

$$
\begin{aligned}
& \dot{\bar{x}}(t)=H\left(I_{N} \otimes A-L_{\sigma(t)} \otimes\left(B K_{\sigma(t)}\right)\right) H^{-1} \bar{x}(t), \\
& \bar{x}(0)=\bar{x}_{0} .
\end{aligned}
$$

Let $\bar{x}=\left[\begin{array}{ll}y^{T} & z^{T}\end{array}\right]^{T}$, where $y=\left[\begin{array}{lll}\bar{x}_{1}^{T} & \ldots & \bar{x}_{N-1}^{T}\end{array}\right]^{T}, z=\bar{x}_{N}$. Then system (9) can be rewritten into two equations:

$$
\begin{aligned}
& \dot{y}=\bar{A}_{\sigma(t)} y, \\
& \dot{z}=\bar{C}_{\sigma(t)} y+A z,
\end{aligned}
$$

where

$$
\begin{aligned}
& \bar{A}_{\sigma(t)}=I_{N-1} \otimes A-\left(\widetilde{H}_{0} L_{\sigma(t)} \widehat{H}_{0}\right) \otimes\left(B K_{\sigma(t)}\right), \\
& \bar{C}_{\sigma(t)}=-\left(\mathbf{1}_{N}^{T} L_{\sigma(t)} \widehat{H}_{0}\right) \otimes\left(B K_{\sigma(t)}\right) .
\end{aligned}
$$

As $\sigma(t)$ is a piecewise constant function that maps into $\mathfrak{M}$, the first equation of system (10) can be expressed by a switched system with $M$ subsystems and a switching signal $\sigma(t)$ :

$$
\dot{y}=\bar{A}_{l} y, \quad l \in \mathfrak{M} .
$$

Definition 5 (see [20]). The equilibrium point $y=0$ of the switched system (12) is said to be exponentially stable under switching signal $\sigma(t)$ if the solution $y(t)$ satisfies

$$
\|y(t)\| \leq \eta\left\|y\left(t_{0}\right)\right\| e^{-\lambda\left(t-t_{0}\right)}, \quad \forall t \geq t_{0}
$$

where constants $\eta \geq 1$ and $\lambda>0$.

The consensus of system (1) via protocol (2) is equivalent to the asymptotic stability of equilibrium point $y=0$ for the switched system (12). Thus, similar to the proof process of Lemma 2 and Theorem 1 in [11], we arrive at the following lemma.

Lemma 6. For given topology set $\left\{\mathcal{N}^{l}, l \in \mathfrak{M}\right\}$ and a switching signal $\sigma(t)$, via protocol (2), system (1) achieves state consensus if and only if the switched system (12) is exponentially stable.

Let $\sigma(t) \equiv l$. From Lemma 6 we can derive a consensus criterion of system (1) under fixed topology $\mathscr{N}^{l}$ as follows.

Corollary 7. $\mathcal{N}^{l}$, the topology of system (1), is consensusable if and only if the matrix $\bar{A}_{l}$ in (12) is Hurwitz stable. 


\section{Main Results}

In this section, based on the dynamic dwell time method, we will analyze a sufficient condition that guarantees the consensus of system (1) under multitype switching topologies.

Suppose topology $\mathcal{N}(t)$ satisfies Assumption 3. From Corollary 7, we obtain that $\bar{A}_{l}, l \in \mathfrak{M}^{-}$in (12) is Hurwitz stable while $\bar{A}_{l}, l \in \mathfrak{M}^{+}$is not Hurwitz stable. Hence, there are positive scalars $\lambda_{l}, l \in \mathfrak{M}$ and positive definite matrices $Q_{l}, l \in \mathfrak{M}$ such that

$$
\begin{aligned}
& \left(\bar{A}_{l}+\lambda_{l} I\right)^{T} Q_{l}+Q_{l}\left(\bar{A}_{l}+\lambda_{l} I\right)<0, \quad l \in \mathfrak{M}^{-}, \\
& \left(\bar{A}_{l}-\lambda_{l} I\right)^{T} Q_{l}+Q_{l}\left(\bar{A}_{l}-\lambda_{l} I\right)<0, \quad l \in \mathfrak{M}^{+} .
\end{aligned}
$$

Define the Lyapunov functions

$$
V_{l}(y)=y^{T} Q_{l} y, \quad y \in \mathbb{R}^{(N-1) n}, l \in \mathfrak{M}
$$

As $\mathcal{N}(t)$ switches among topology set $\mathscr{N} \mathscr{A}=\mathscr{N} \mathscr{C} \cup \mathcal{N} \mathscr{U}$. Without loss of generality, suppose system (1) firstly runs under several consensusable topologies in succession, which are denoted by a consensusable subgroup $\widetilde{\mathcal{N}}_{k}, k=1,2, \ldots$ and then follow with several unconsensusable topologies one after another, which is denoted as unconsensusable subgroup ${\widetilde{N} \mathscr{U}_{k}}, k=1,2, \ldots$, and that cycle repeats. Hence, we have an assumption about switching signal $\sigma(t)$ as follows.

Assumption 8 . The consensusable subgroup $\widetilde{N \mathscr{C}}_{k}, k=$ $1,2, \ldots$ is first activated and then follows with the uncon-

\begin{tabular}{|c|c|}
\hline subgroup & switching instants \\
\hline$\ldots$ & .. \\
\hline$\overbrace{\mathcal{N} \mathscr{C}_{k-1}}$ & $t_{(k-1)^{-}}, t_{(k-1)^{-}+1}, \ldots, t_{(k-1)^{-}+l_{(k-1)^{-}}}$ \\
\hline$\overbrace{\mathscr{N} U_{k-1}}$ & $t_{(k-1)^{+}}, t_{(k-1)^{+}+1}, \ldots, t_{(k-1)^{+}+l_{(k-1)^{+}}}$ \\
\hline$\overbrace{\mathcal{N} \mathscr{C}_{k}}$ & $t_{k^{-}}, t_{k^{-}+1}, \ldots, t_{k^{-}+l_{k^{-}}}$ \\
\hline$\overbrace{\mathcal{N} \mathcal{U}_{k}}$ & $t_{k^{+}}, t_{k^{+}+1}, \ldots, t_{k^{+}+l_{k^{+}}}$ \\
\hline$\cdots$ & $\cdots$, \\
\hline
\end{tabular}
sensusable subgroup $\widetilde{\mathcal{N}}_{k}$, and that cycle repeats. Switching signal $\sigma(t)$ has a sequence of switching instants as follows:

where $l_{k^{-}}=1,2, \ldots$ and $l_{k^{+}}=0,1,2, \ldots$.

Remark 9. From Assumption 8, we know that, for any positive number $T$, there exists interval $\left[t_{k^{-}}, t_{k^{-}+1}\right) \in[T, \infty)$ such that a consensusable topology is activated.

Let $l^{-}$be the index of the former consensusable topology of $\mathcal{N}^{l}, l \in \mathfrak{M}^{-} . t^{-}\left(t^{+}\right)$is the left (right) limit of $t$.

Theorem 10. Assume that topology $\mathcal{N}(t)$ of system (1) satisfies Assumption 3 and the switching signal $\sigma(t)$ satisfies Assumption 8. System (1) can achieve consensus via protocol (2) with the switching signal $\sigma(t)$ if the corresponding dwell time $\tau_{l}$ of $\mathscr{N}^{l}, l=\sigma\left(t_{k^{-}}\right) \in \mathfrak{M}^{-}$for system (1) satisfies

$$
\tau_{l}\left(t_{k^{-}}\right)> \begin{cases}\left(2 \lambda_{l}\right)^{-1} \bar{\Delta}_{l}\left(y\left(t_{k^{-}}\right)\right), & \text {if } \bar{\delta}_{l}\left(y\left(t_{k^{-}}\right)\right)>0 \\ 0, & \text { if } \bar{\delta}_{l}\left(y\left(t_{k^{-}}\right)\right) \leq 0,\end{cases}
$$

where

$$
\begin{aligned}
& \bar{\delta}_{l}\left(y\left(t_{k^{-}}\right)\right)=V_{l}\left(y\left(t_{k^{-}}\right)\right)-V_{l^{-}}\left(y\left(t_{(k-1)^{+}}\right)\right), \\
& \bar{\Delta}_{l}\left(y\left(t_{k^{-}}\right)\right)=\ln V_{l}\left(y\left(t_{k^{-}}\right)\right)-\ln V_{l^{-}}\left(y\left(t_{(k-1)^{+}}\right)\right) .
\end{aligned}
$$

Proof. First, we prove that switched system (12) is asymptotic stable. It contains three steps as follows.

Step 1. Condition (17) implies

$$
V\left(y\left(t_{k^{-}+1}^{-}\right)\right)<V\left(y\left(t_{(k-1)^{+}}^{-}\right)\right), \quad k=1,2, \ldots
$$

Considering the Lyapunov functions $V_{l}(y)$ in (15), there are

$$
\alpha_{1}\|y\|^{2} \leq V_{l}(y) \leq \alpha_{2}\|y\|^{2}, \quad l \in \mathfrak{M},
$$

where $\alpha_{1}=\min _{l \in \mathfrak{M}} \lambda_{\text {min }}\left(Q_{l}\right)$ and $\alpha_{2}=\max _{l \in \mathfrak{M}} \lambda_{\text {max }}\left(Q_{l}\right)$. Let $\bar{\lambda}=\max _{l \in \mathfrak{M}^{+}} \lambda_{l}$ and $\underline{\lambda}=\min _{l \in \mathfrak{M}^{-}} \lambda_{l}$. From (14), we obtain

$$
\dot{V}_{l}(y(t))< \begin{cases}-2 \lambda_{l} V_{l}(y(t)), & l \in \mathfrak{M}^{-}, \\ 2 \bar{\lambda} V_{l}(y(t)), & l \in \mathfrak{M}^{+}\end{cases}
$$

Hence, for $t \in\left[t_{k^{-}}, t_{k^{-}+1}\right), k=1,2, \ldots$, there is

$$
V(y(t))=e^{-2 \lambda_{l}\left(t-t_{k^{-}}\right)} V\left(y\left(t_{k^{-}}^{+}\right)\right) .
$$

Let $t=t_{k^{-}+1}^{-}$, and, from (21), we derive

$$
\begin{aligned}
& V\left(y\left(t_{k^{-}+1}^{-}\right)\right)<e^{-2 \lambda_{l}\left(t_{k^{-}+1}-t_{k^{-}}\right)} V\left(y\left(t_{k^{-}}^{+}\right)\right) \\
& =e^{-2 \lambda_{l} \tau\left(t_{k^{-}}\right)} \frac{V\left(y\left(t_{k^{-}}^{+}\right)\right)}{V\left(y\left(t_{(k-1)^{+}}^{-}\right)\right)} V\left(y\left(t_{(k-1)^{+}}^{-}\right)\right) \\
& =e^{-2 \lambda_{l} \tau\left(t_{k^{-}}\right)} \frac{V_{l}\left(y\left(t_{k^{-}}\right)\right)}{V_{l^{-}}\left(y\left(t_{(k-1)^{+}}\right)\right)} V\left(y\left(t_{(k-1)^{+}}^{-}\right)\right) \\
& =e^{\phi} V\left(y\left(t_{(k-1)^{+}}^{-}\right)\right),
\end{aligned}
$$

where $\phi=-2 \lambda_{l} \tau\left(t_{k^{-}}\right)+\ln V_{l}\left(y\left(t_{k^{-}}\right)\right)-\ln V_{l^{-}}\left(y\left(t_{(k-1)^{+}}\right)\right)$. Thus, formula (19) is available. 
Step 2. Without loss of generality, assume that $x\left(t_{1^{-}}\right)$is the initial state. According to (20), we obtain

$$
V\left(y\left(t_{k^{+}}^{+}\right)\right) \leq \alpha_{2}\left\|y\left(t_{k^{+}}\right)\right\| \leq \frac{\alpha_{2}}{\alpha_{1}} V\left(y\left(t_{k^{+}}^{-}\right)\right) .
$$

By (21), for $t \in\left[t_{k^{+}}, t_{(k+1)^{-}}\right)$, we have

$$
\begin{aligned}
& V(y(t))<e^{2 \bar{\lambda}\left(t-t_{k^{+}}\right)} \frac{\alpha_{2}}{\alpha_{1}} V\left(y\left(t_{k^{+}}^{-}\right)\right), \\
& \forall t \in\left[t_{k^{+}}, t_{k^{+}+1}\right) ; \\
& V(y(t))<e^{2 \bar{\lambda}\left(t-t_{k^{+}}\right)}\left(\frac{\alpha_{2}}{\alpha_{1}}\right)^{2} V\left(y\left(t_{k^{+}}^{-}\right)\right), \\
& \forall t \in\left[t_{k^{+}+1}, t_{k^{+}+2}\right) ; \\
& V(y(t))<e^{2 \bar{\lambda}\left(t-t_{k^{+}}\right)}\left(\frac{\alpha_{2}}{\alpha_{1}}\right)^{l_{k^{+}+1}} V\left(y\left(t_{k^{+}}^{-}\right)\right) \\
& \forall t \in\left[t_{k^{+}+l_{k^{+}}}, t_{(k+1)^{-}}\right) .
\end{aligned}
$$

It concludes that

$$
V(y(t))<e^{2 \bar{\lambda}\left(t-t_{k^{+}}\right)} h_{k} V\left(y\left(t_{k^{+}}^{-}\right)\right), \quad t \in\left[t_{k^{+}}, t_{(k+1)^{-}}\right),
$$

where $h_{k}=\max _{m=1, \ldots, l_{k^{+}+1}}\left\{\left(\alpha_{2} / \alpha_{1}\right)^{m}\right\}$. By using (19), we obtain

$$
\begin{aligned}
V(y(t)) & <e^{2 \bar{\lambda}\left(t-t_{k^{+}}\right)} h_{k} V\left(y\left(t_{k^{+}}^{-}\right)\right)<\cdots \\
& <e^{2 \bar{\lambda}\left(t_{(k+1)^{-}}-t_{k^{+}}\right)} \prod_{s=1}^{k} h_{s} \beta V\left(y\left(t_{1^{-}}\right)\right) .
\end{aligned}
$$

Thus, for any $\varepsilon>0$, there exists $\delta_{1}=$ $\left(\alpha_{1}^{-1} \alpha_{2} e^{2 \bar{\lambda}\left(t_{(k+1)^{-}}-t_{k^{+}}\right)} \prod_{s=1}^{k} h_{s} \beta\right)^{-1 / 2} \varepsilon$, such that $\|y(t)\|<\varepsilon$ for all $t \in\left[t_{1^{-}}, \infty\right)$, whenever $\left\|y\left(t_{1}\right)\right\|<\delta_{2}$.

Step 3. When $t \in\left[t_{k^{-}+1}, t_{k^{+}}\right)$, we get

$$
\begin{gathered}
V(y(t))<e^{-2 \underline{\lambda}\left(t-t_{k^{-}+1}\right)} \alpha_{2} \alpha^{-1} V\left(y\left(t_{k^{-}+1}^{-}\right)\right), \\
\forall t \in\left[t_{k^{-}+1}, t_{k^{-}+2}\right) ; \\
V(y(t))<e^{-2 \underline{\lambda}\left(t-t_{k^{-}+1}\right)} \alpha_{2}^{2} \alpha^{-2} V\left(y\left(t_{k^{-}+1}^{-}\right)\right), \\
\forall t \in\left[t_{k^{-}+2}, t_{k^{-}+3}\right) ; \\
\vdots \\
V(y(t))<e^{-2 \underline{\lambda}\left(t-t_{k^{-}+1}\right)} \alpha_{2}^{l_{k^{-}}} \alpha^{-l_{k^{-}}} V\left(y\left(t_{k^{-}+1}^{-}\right)\right), \\
\forall t \in\left[t_{k+l_{k^{-}}}, t_{k^{+}}\right) .
\end{gathered}
$$

In summary,

$$
V(y(t))<\bar{h}_{k} V\left(\mathrm{y}\left(t_{k^{-}+1}^{-}\right)\right), \quad t \in\left[t_{k^{-}+1}, t_{k^{+}}\right),
$$

where $\bar{h}_{k}=\max _{r=1, \ldots, l_{k^{-}}}\left\{\left(\alpha_{2} / \alpha_{1}\right)^{r}\right\}$. Consider

$$
\begin{aligned}
V(y(t)) & \left.<e^{-2 \underline{\lambda}\left(t-t_{k^{-}+1}^{-}\right.}\right) \bar{h}_{k} V\left(y\left(t_{k^{-}+1}^{-}\right)\right)<\cdots \\
& <\prod_{s=1}^{k} \bar{h}_{s} \beta V\left(y\left(t_{1^{-}}\right)\right)
\end{aligned}
$$

where $\beta$ is a positive constant.

Therefore, for any $\varepsilon>0$, there exist $\delta_{2}=$ $\left(\alpha_{1}^{-1} \alpha_{2} \prod_{s=1}^{k} \bar{h}_{s} \beta\right)^{-1 / 2} \varepsilon$ such that $\|y(t)\|<\varepsilon$ for all $t \in\left[t_{1^{-}}, \infty\right)$, whenever $\left\|y\left(t_{1^{-}}\right)\right\|<\delta_{1}$.

Hence, for any $t>0$ and $\varepsilon>0$, there exist $\delta_{2}=$ $\max \left(\delta_{1}, \delta_{2}\right)$ such that $\|y(t)\|<\varepsilon$. Consequently, the switched system (12) is asymptotic stable.

From Lemma 6 we know that system (1) achieves consensus. The proof is complete.

Remark 11. Let $\mu=\sup _{y \in \mathbb{R}^{n(N-1)}}\left\{\mu \| \mu=V_{l}\left(y\left(t_{k^{-}}\right)\right) /\right.$ $\left.V_{l^{-}}\left(y\left(t_{(k-1)^{+}}\right)\right) ; l, l^{-} \in \mathfrak{M}^{-}\right\}, \lambda^{-}=\min _{l \in \mathfrak{M}^{-}}\left\{\lambda_{l}\right\}, \lambda^{+}=$ $\max _{l \in \mathfrak{M}^{+}}\left\{\lambda_{l}\right\}$, and choose $\lambda^{*} \in\left(\lambda, \lambda^{-}\right)$. It can be seen that the condition in Theorem 10 implies both $\tau>(\ln \mu) / \lambda$ and time ratio condition $\inf _{t \geq 0}\left(T^{-}(t) / T^{+}(t)\right) \geq\left(\lambda^{+}+\lambda^{*}\right) /\left(\lambda^{-}-\lambda^{*}\right)$, where $T^{-}(t)$ (resp., $T^{+}(t)$ ) stands for the total activation time of system (1) under consensusable (resp., unconsensusable) topologies. Theorem 2 in [16] is a special case of Theorem 10.

Remark 12. When unconsensusable topology set $\mathscr{N} \mathcal{U}=\emptyset$, that is, $\mathcal{N}(t)$ switches in consensusable topology set $\mathscr{N} \mathscr{A}=$ $\mathscr{N} \mathscr{C}$, it can be seen that Theorem 1 in [19] is only a special case of Theorem 10.

\section{Simulation Example}

In this section, we give a numerical simulation to illustrate the obtained result. Consider a multiagent system with 6 agents:

$$
\dot{x}_{i}(t)=\left[\begin{array}{ll}
0 & 1 \\
0 & 0
\end{array}\right] x_{i}(t)+\left[\begin{array}{l}
0 \\
1
\end{array}\right] u_{i}(t), \quad i=1, \ldots, 6
$$

with protocol

$$
u_{i}(t)=\left[\begin{array}{ll}
1 & 1.2
\end{array}\right] \sum_{j \in N_{i}^{\sigma(t)}} w_{i j}^{\sigma(t)}\left(x_{j}(t)-x_{i}(t)\right),
$$

where $x_{i}(t)=\left[x_{i 1}(t), x_{i 2}(t)\right]^{T}$.

Figure 1 shows four different directed topologies $\mathcal{N}^{l}$ : $l=1,2,3,4$. The weight of each edge is 1 . From Definition 2 and Corollary 7, it derives that $\mathscr{N}^{1}$ and $\mathscr{N}^{3}$ are consensusable topologies while $\mathscr{N}^{2}$ and $\mathscr{N}^{4}$ are unconsensusable topologies. Consider switching topologies with a periodic switch sequence $S: \mathscr{N}^{1} \rightarrow \mathcal{N}^{2} \rightarrow \mathcal{N}^{3} \rightarrow \mathcal{N}^{4} \rightarrow \mathcal{N}^{1} \cdots$.

Next, we compare the consequences of two types of switching signals which are correspondingly based on the dynamic dwell time method (DDTM) in this paper and on the average dwell time method (ADTM) in [16].

By use of the ADTM in [16], we obtain that if the switching signals of topologies satisfy both average dwell 

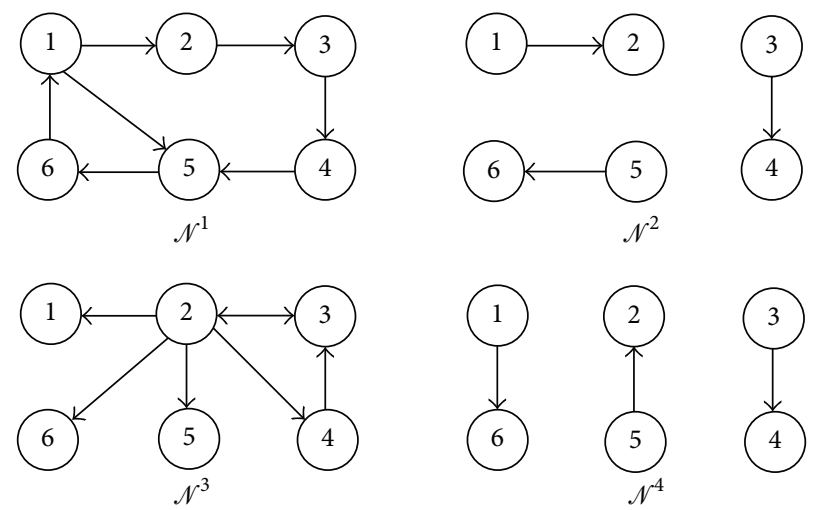

FIGURE 1: Four possible topologies of system (31).
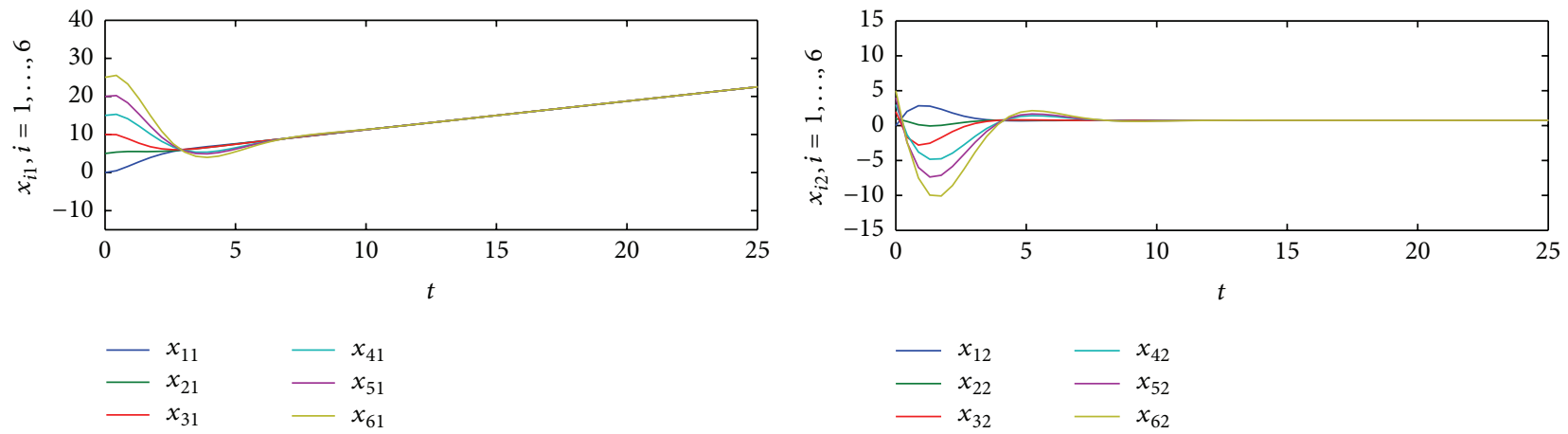

FIGURE 2: State trajectories of system (31) with $\sigma_{a}(t)$.

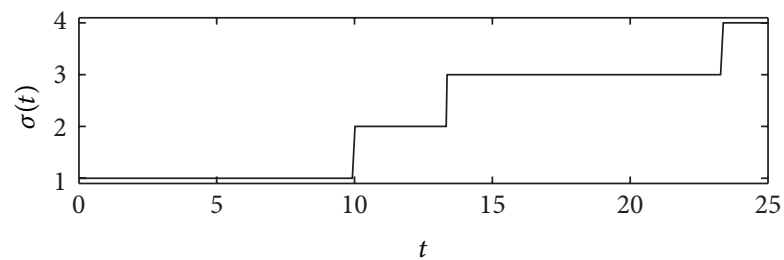

Figure 3: A switching signal $\sigma_{a}(t)$ of $\mathscr{N}^{1}, \mathscr{N}^{2}, \mathscr{N}^{3}, \mathscr{N}^{4}$.

time condition $\tau_{a} \geq 6.6776$ and time ratio condition $\inf _{t \geq 0} T^{-}(t) / T^{+}(t) \geq 3$, via protocol (32), system (31) can achieve consensus under switching topology. Figure 2 depicts the state trajectory of each state component of the six agents under the aforementioned switching topologies and a switching signal $\sigma_{a}(t)$ in Figure 3 with the switching sequence $S$.

By using the DDTM (17), we can obtain the dynamic dwell time $\tau_{l}\left(t_{k}\right), l=1,2,3,4, k=1,2, \ldots$, that evolves on the topologies $\mathcal{N}^{l}, l=1,2,3$, . If switching signals of topology satisfy dynamic dwell time condition, via protocol (32), system (31) can achieve consensus under switching topologies. Part values of the minimum dwell time of a switching signal are shown in Table 1. Choose dwell time $\tau_{l}\left(t_{k}\right)+0.2$; Figure 4 gives the state trajectory of each state
TABLE 1: Part values of the minimum dwell time $\tau_{l}\left(t_{k}\right)$.

\begin{tabular}{ccccc}
\hline Topology & $\mathcal{N}^{1}$ & $\mathscr{N}^{2}$ & $\mathscr{N}^{3}$ & $\mathscr{N}^{4}$ \\
\hline \multirow{6}{*}{$\tau_{l}\left(t_{k}\right)$} & 0 & 0.1903 & 0 & 0.284 \\
& 1.4086 & 0.0380 & 0 & 0.0270 \\
& 0.7497 & 0.2654 & 0 & 0.3896 \\
& 0.9814 & 0.4671 & 0.2749 & 0.0650 \\
& 0.5577 & 0.2845 & 0.0557 & 0.2347 \\
& 0.5707 & 0.0060 & 0 & 0.1686 \\
& $\ldots$ & $\ldots$ & $\ldots$ & $\ldots$ \\
\hline
\end{tabular}

component of the six agents under the aforementioned switching topologies and a switching signal $\sigma_{d}(t)$ in Figure 5 with the switching sequence $S$.

What is shown in Figures 2 and 4 indicates that, for the same dynamic, topologies, switching sequence $S$, protocol, and initial states, system (31) can achieve consensus by the use of switching signals based on average and dynamic dwell time methods. However, switching signal $\sigma_{a}(t)$ in Figure 3 switches 4 times within 25 seconds; in contrast, switching signal $\sigma_{d}(t)$ in Figure 5 switches 68 times within 25 seconds. Based on the above analysis, DDTM (17) is less conservative and provides more flexible switching signals in practical ways for the LMASs to achieve consensus. 

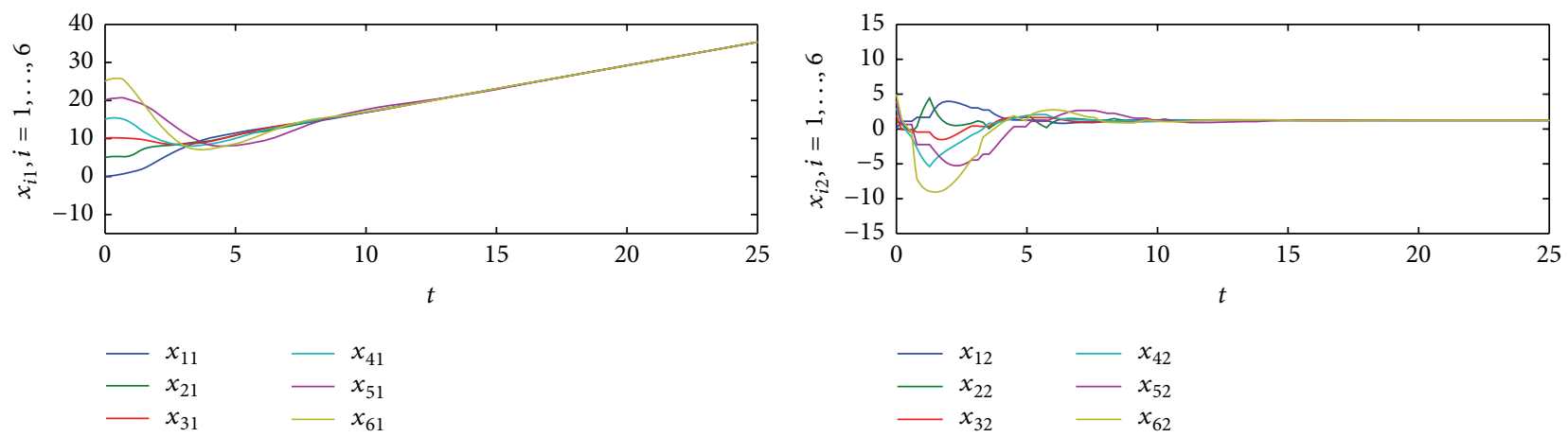

Figure 4: State trajectories of system (31) with $\sigma_{d}(t)$.

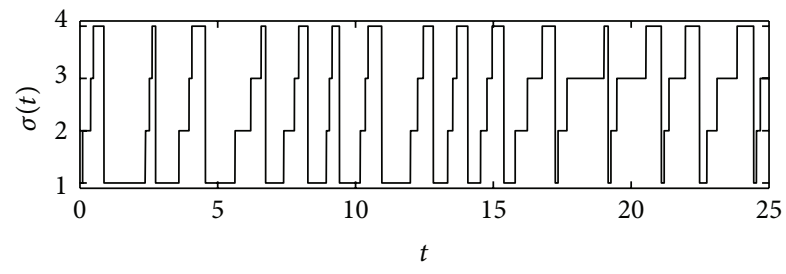

Figure 5: A switching signal $\sigma_{d}(t)$ of $\mathscr{N}^{1}, \mathscr{N}^{2}, \mathscr{N}^{3}, \mathscr{N}^{4}$.

\section{Conclusion}

The consensus problem has been studied for high-order LMASs under multitype communication topologies that include both consensusable and unconsensusable topologies. By using a linear transformation, a lemma has been presented which analyzes the equivalence relation between the consensus of LMASs and the asymptotic stability of a corresponding system. Based on the dynamic dwell time method, a switching rule has been characterized. With a class of switching signals which satisfies this switching rule, the consensus of LMASs can be achieved under multitype switching topologies. Finally, the effectiveness of the proposed theory has been illustrated by a numerical example.

\section{Conflict of Interests}

The authors declare that there is no conflict of interests regarding the publication of this paper.

\section{Acknowledgment}

This work is partially supported by Natural Science Foundation of China (61273006, 61511130044, and 61573030).

\section{References}

[1] R. Olfati-Saber, "Flocking for multi-agent dynamic systems: algorithms and theory," IEEE Transactions on Automatic Control, vol. 51, no. 3, pp. 401-420, 2006.

[2] H. Yang, C. Wang, and F. Zhang, "Robust geometric formation control of multiple autonomous underwater vehicles with time delays," in Proceedings of the American Control Conference (ACC '13), pp. 1380-1385, IEEE, Washington, DC, USA, June 2013.
[3] W. Ren and R. W. Beard, Distributed Consensus in MultiVehicle Cooperative Control: Theory and Applications, Springer, London, UK, 2007.

[4] R. Olfati-Saber and P. Jalalkamali, "Coupled distributed estimation and control for mobile sensor networks," IEEE Transactions on Automatic Control, vol. 57, no. 10, pp. 2609-2614, 2012.

[5] R. Olfati-Saber and R. M. Murray, "Consensus problems in networks of agents with switching topology and time-delays," IEEE Transactions on Automatic Control, vol. 49, no. 9, pp. 15201533, 2004.

[6] W. Ren and R. W. Beard, "Consensus seeking in multiagent systems under dynamically changing interaction topologies," IEEE Transactions on Automatic Control, vol. 50, no. 5, pp. 655661, 2005.

[7] W. Ren, R. W. Beard, and E. M. Atkins, "Information consensus in multivehicle cooperative control," IEEE Control Systems Magazine, vol. 27, no. 2, pp. 71-82, 2007.

[8] F. Xiao and L. Wang, "Consensus problems for high dimensional multi-agent systems," IET Control Theory \& Applications, vol. 1, no. 3, pp. 830-837, 2007.

[9] C.-Q. Ma and J.-F. Zhang, "Necessary and sufficient conditions for consensusability of linear multi-agent systems," IEEE Transactions on Automatic Control, vol. 55, no. 5, pp. 1263-1268, 2010.

[10] W. Yu, G. Chen, W. Ren, J. Kurths, and W. X. Zheng, "Distributed higher order consensus protocols in multiagent dynamical systems," IEEE Transactions on Circuits and Systems. I. Regular Papers, vol. 58, no. 8, pp. 1924-1932, 2011.

[11] Y. Z. Chen, Y. R. Ge, and Y. X. Zhang, "Partial stability approach to consensus problem of linear multiagent systems," Acta Automatica Sinica, vol. 40, no. 11, pp. 2573-2584, 2014.

[12] J. Wang, D. Cheng, and X. Hu, "Consensus of multi-agent linear dynamic systems," Asian Journal of Control, vol. 10, no. 2, pp. 144-155, 2008.

[13] Y. Su and J. Huang, "Stability of a class of linear switching systems with applications to two consensus problems," IEEE Transactions on Automatic Control, vol. 57, no. 6, pp. 1420-1430, 2012.

[14] L. Cheng, H. Wang, Z.-G. Hou, and M. Tan, "Reaching a consensus in networks of high-order integral agents under switching directed topologies," International Journal of Systems Science, 2014.

[15] T. Liu, J. Zhao, and D. J. Hill, "Exponential synchronization of complex delayed dynamical networks with switching topology," IEEE Transactions on Circuits and Systems. I. Regular Papers, vol. 57, no. 11, pp. 2967-2980, 2010. 
[16] Y. Chen, Y. Zhang, Z. He, and Y. Ge, "Average dwell-time conditions of switching information topologies for consensus of linear multi-agent systems," in Proceedings of the 32th IEEE Chinese Control Conference (CCC '13), pp. 7115-7120, Xian, China, July 2013.

[17] Y. R. Ge, Y. Z. Chen, and Y. X. Zhang, "Average dwell-time conditions for consensus of discrete-time linear multi-agent systems with switching topologies and time-varying delays," Acta Automatica Sinica, vol. 40, no. 11, pp. 2609-2617, 2014.

[18] Z. R. Xiang and W. M. Xiang, "Stability analysis of switched systems under dynamical dwell time control approach," International Journal of Systems Science, vol. 40, no. 4, pp. 347-355, 2009.

[19] Z. Yaxiao, C. Yangzhou, and A. Y. Aleksandrov, "Dynamic dwell-time condition of switching information topology for consensus of linear multi-agent systems," in Proceedings of the 34th Chinese Control Conference (CCC '15), pp. 6918-6923, Hangzhou, China, July 2015.

[20] D. Liberzon, Switching in Systems and Control, Springer, Boston, Mass, USA, 2003. 


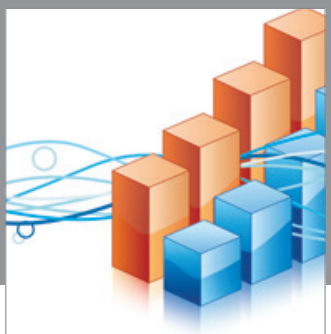

Advances in

Operations Research

vatem alat4

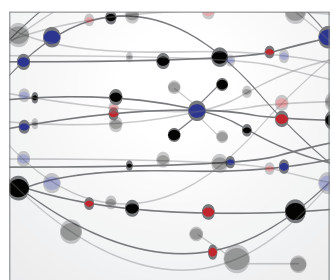

\section{The Scientific} World Journal
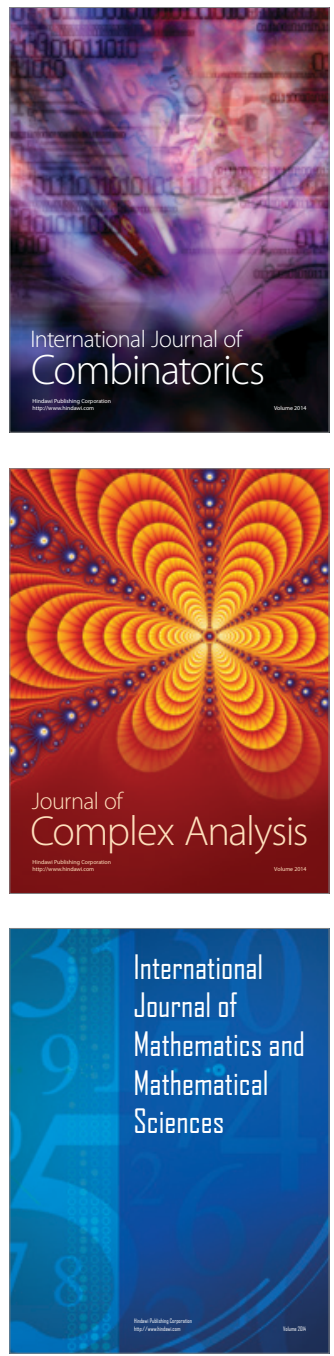
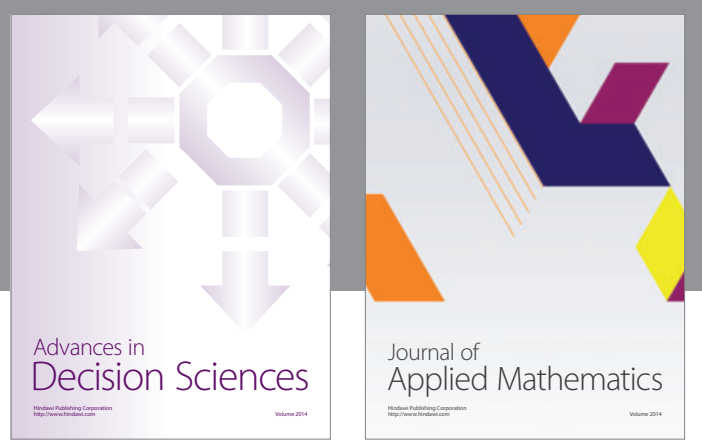

Algebra

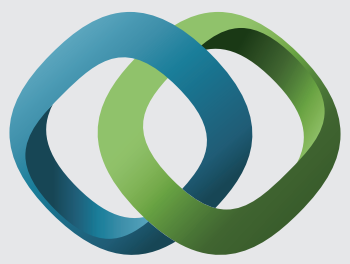

\section{Hindawi}

Submit your manuscripts at

http://www.hindawi.com
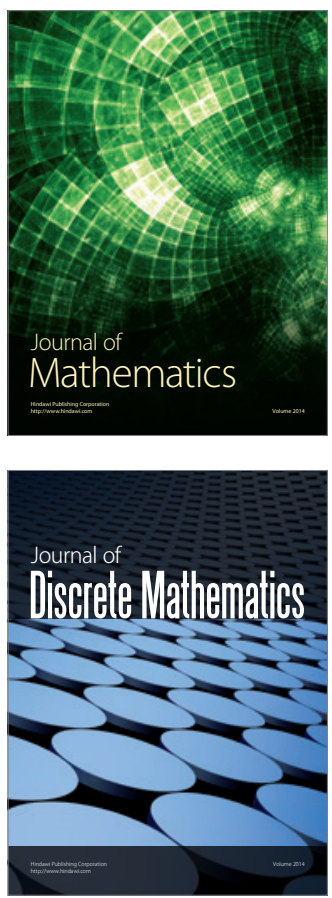

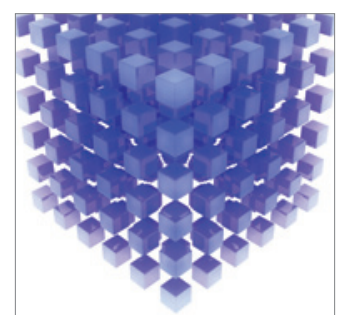

Mathematical Problems in Engineering
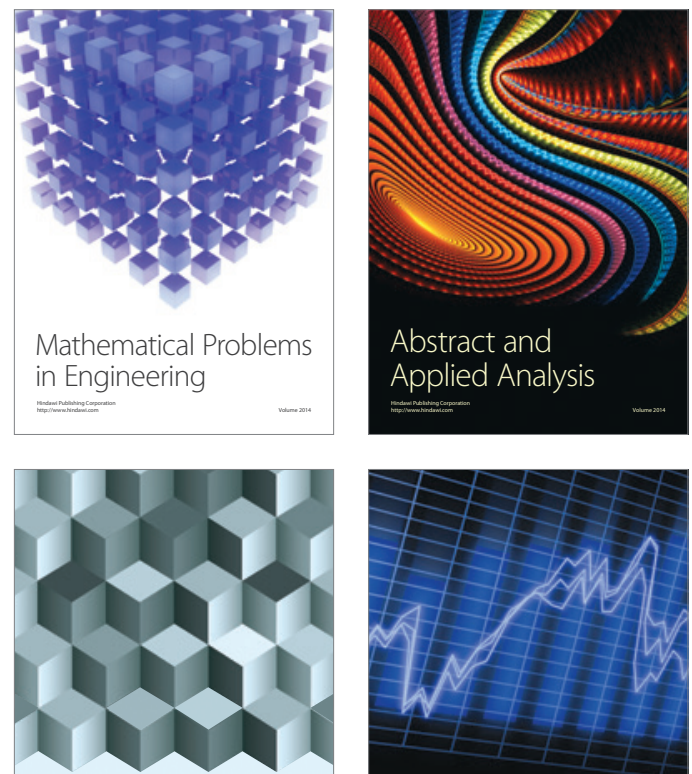

Journal of

Function Spaces

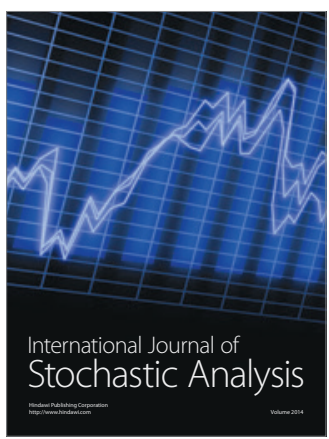

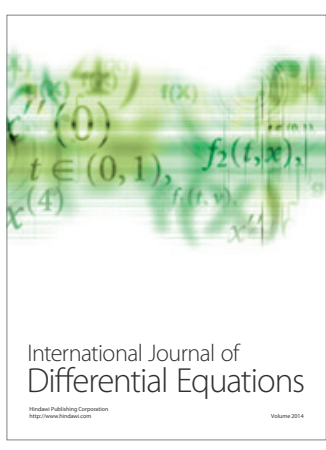
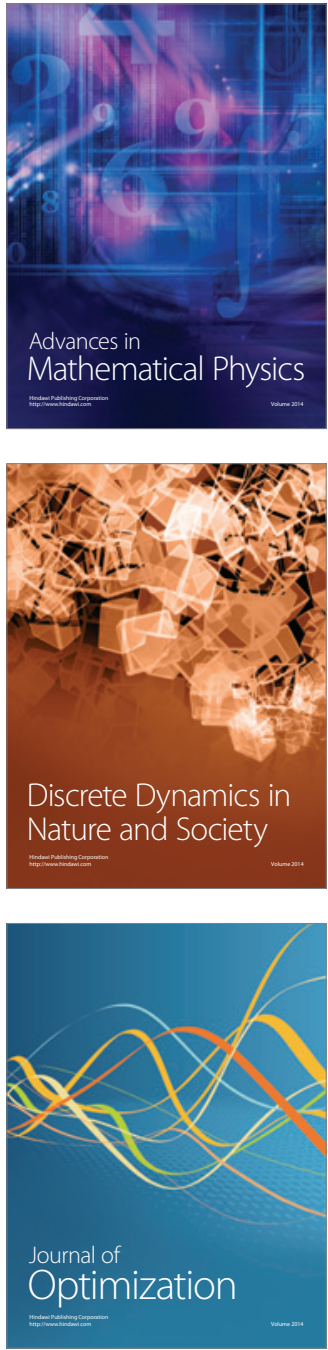